\title{
Quantum-corrected black hole thermodynamics to all orders in the Planck length
}

\author{
Khireddine Nouicer* \\ Laboratory of Theoretical Physics and Department of Physics, \\ Faculty of Sciences, University of Jijel \\ Bp 98, Ouled Aissa, 18000 Jijel, Algeria.
}

\begin{abstract}
We investigate the effects to all orders in the Planck length from a generalized uncertainty principle (GUP) on black holes thermodynamics. We calculate the corrected Hawking temperature, entropy, and examine in details the Hawking evaporation process. As a result, the evaporation process is accelerated and the evaporation end-point is a zero entropy, zero heat capacity and finite non zero temperature black hole remnant (BHR). In particular we obtain a drastic reduction of the decay time, in comparison with the result obtained in the Hawking semi classical picture and with the GUP to leading order in the Planck length.
\end{abstract}

PACS: 04.60.-m, 05.70.-a

Key Words: Quantum Gravity, Generalized Uncertainty Principle, Thermodynamics of Black Holes

\section{Introduction}

Recently a great interest has been devoted to the study of the effects of generalized uncertainty principles (GUPs) and modified dispersion relations (MDRs) on black holes thermodynamics. The concepts of GUPs and MDRs originates from several studies in string theory approach to quantum gravity [1-4], loop quantum gravity [5], noncommutative space-time algebra [6 - 8] and black holes gedanken experiments [9 - 10]. All these approaches indicate that the standard Heisenberg uncertainty principle must be generalized to incorporate additional uncertainties when quantum gravitational effects are taken into account. Actually it is believed that any

\footnotetext{
*Email: khnouicer@mail.univ-jijel.dz / khnouicer@yahoo.fr
} 
promising candidate for a quantum theory of gravity must include the GUPs and/or MDRs as central ingredients.

The main consequence of the GUP is the appearance of a minimal length scale of the order of the Planck length which cannot be probed, providing a natural UV cut-off, and thus corrections to black holes thermodynamic parameters are expected at the Planck scale.

The consequences of GUPs and/or MDRs on black holes thermodynamics have been considered intensively in the recent literature on the subject [11 - 16]. Notably, it has been shown that GUP prevents black holes from complete evaporation, exactly like the standard Heisenberg principle prevents the hydrogen atom from total collapse [17]. Then at the final stage of the Hawking radiation process of a black hole, a inert black hole remnant (BHR) continue to exist with zero entropy, zero heat capacity and a finite non zero temperature. The inert character of the BHR, besides gravitational interactions, makes this object a serious candidate to explain the nature of dark matter [18, 19]. On the other hand, a particular attention has been also devoted to the computation of the entropy of a black hole and the sub-leading logarithmic correction [20 - 34].

All the above studies have been performed with a GUP to leading order in the Planck length. However, recent generalization of the GUP induces quantitative corrections to the entropy and then influences the evaporation phase of the black hole [35]. Besides this growing interest in quantum gravity phenomenology, a intense activity is actually devoted to possible production of black holes at particle colliders [36, 37] and in ultrahigh energy cosmic ray (UHECR) airshowers [38, 39]. The next generation of particle colliders are planned to reach a $c-m$ energy of the order of few $T e V$, a scale at which the complete evaporation of $\mathrm{BH}$ is expected to end, leaving up in a scenario with GUP a inert BHR. Then, it is phenomenologically relevant, to obtain the corrections to BH thermodynamic parameters in the framework of a GUP beyond the leading order in the Planck length.

In this paper we discuss the effects, brought by a generalization of the GUP to all orders in the Planck length, on thermodynamic parameters of the Schwarzschild black hole . Hereafter, we refer to this version of GUP as GUP*.

The organization of this work is as follows. In section 2, we introduce a deformed position and momentum operators algebra leading to $\mathrm{GUP}^{*}$ and examine its various implications. In section 3, the Hawking temperature and entropy are computed and the departures from the standard case shown. In section 4, we calculate the deviation from the standard Stefan-Boltzmann law of the black body radiation spectrum and investigate the Hawking evaporation process of black holes by a calculation of the evaporation rate, the decay time and the heat capacity. Finally we compare our results with the ones obtained in the context of the GUP to leading order in the Planck length commonly used in the literature. Our conclusions are summarized in the last section. 


\section{Generalized uncertainty principle}

Loop quantum gravity and string theory approach to quantum gravity predict slight deviations in the laws describing photons propagation in vacuum. It is expected that these effects, leading to a modified dispersion relation (MDR), could be amplified by cosmological distances and then become observables [40]. On the other hand, quantum gravity phenomenology has been tackled within effective models based on MDRs and/or GUPs and containing the minimal length as a natural UV cut-off. Recently the relation between these approaches has been clarified and established [41].

The idea of a minimal length can be modelled in terms of a quantized space-time and goes back to the early days of quantum field theory [42] (see also [40 - 43] ). An other approach is to consider deformations to the standard Heisenberg algebra [7, 8], which lead to generalized uncertainty principles. In this section we follow the latter approach and exploit results recently obtained. Indeed, it has been shown in the context of canonical noncommutative field theory in the coherent states representation [47] and field theory on non-anticommutative superspace [48, 49], that the Feynman propagator display an exponential UV cut-off of the form $\exp \left(-\eta p^{2}\right)$, where the parameter $\eta$ is related to the minimal length. This framework has been further applied, in series of papers [50], to the black hole evaporation process.

At the quantum mechanical level, the essence of the UV finiteness of the Feynman propagator can be also captured by a non linear relation, $p=f(k)$, between the momentum and the wave vector of the particle [41]. This relation must be invertible and has to fulfill the following requirements:

1. For smaller energies than the cut-off the usual dispersion relation is recovered.

2. For large energies, the wave vector asymptotically reaches the cut-off.

In this case, the usual momentum measure $d^{n} p$ is deformed and becomes $d^{n} p \prod_{i} \frac{\partial k_{i}}{\partial p_{j}}$. In the following, we will restrict ourselves to the isotropic case and work with one space-like dimension. Following [47, 49] and setting $\eta=\frac{\alpha^{2} L_{P l}^{2}}{\hbar^{2}}$ we have

$$
\frac{\partial p}{\partial k}=\hbar \exp \left(\frac{\alpha^{2} L_{P l}^{2}}{\hbar^{2}} p^{2}\right),
$$

where $\alpha$ is a dimensionless constant of order one.

From Eq.(11) we obtain the dispersion relation

$$
k(p)=\frac{\sqrt{\pi}}{2 \alpha L_{P l}} \operatorname{erf}\left(\frac{\alpha L_{P l}}{\hbar} p\right),
$$

from which we have the following minimum Compton wavelength

$$
\lambda_{0}=4 \sqrt{\pi} \alpha L_{P l}
$$


Let us show that these results can be obtained from the following representation of the position and momentum operators

$$
X=i \hbar \exp \left(\frac{\alpha^{2} L_{P l}^{2}}{\hbar^{2}} P^{2}\right) \partial_{p} \quad P=p .
$$

The corrections to the standard Heisenberg algebra become effective in the so-called quantum regime where the momentum and length scales are of the order of the Planck mass $M_{P l}$ and of the Planck length $L_{P l}$ respectively.

The hermiticity condition of the position operator implies the following modified completeness relation

$$
\int d p e^{-\frac{\alpha^{2} L_{P l}^{2}}{\hbar^{2}} p^{2}}|p\rangle\langle p|=1
$$

and modified scalar product

$$
\left\langle p \mid p^{\prime}\right\rangle=e^{\frac{\alpha^{2} L_{\mathrm{P} 1}^{2}}{\hbar^{2}} p^{2}} \delta\left(p-p^{\prime}\right) .
$$

From Eq.(5) we observe that we have reproduced the Gaussian damping factor in the Feynman propagator [47, 49]. The algebra defined by Eq. (44) leads to the following generalized commutator and generalized uncertainty principle $\left(\mathrm{GUP}^{*}\right)$

$$
[X, P]=i \hbar \exp \left(\frac{\alpha^{2} L_{P l}^{2}}{\hbar^{2}} P^{2}\right), \quad(\delta X)(\delta P) \geq \frac{\hbar}{2}\left\langle\exp \left(\frac{\alpha^{2} L_{P l}^{2}}{\hbar^{2}} P^{2}\right)\right\rangle .
$$

In order to investigate the quantum implications of this deformed algebra, we consider the saturate GUP* and solve for $(\delta P)$. Using the property $\left\langle P^{2 n}\right\rangle \geq\left\langle P^{2}\right\rangle^{n}$ and $(\delta P)^{2}=\left\langle P^{2}\right\rangle-\langle P\rangle^{2}$ the saturate $\mathrm{GUP}^{*}$ is then given by

$$
(\delta X)(\delta P)=\frac{\hbar}{2} \exp \left(\frac{\alpha^{2} L_{P 1}^{2}}{\hbar^{2}}\left((\delta P)^{2}+\langle P\rangle^{2}\right)\right) .
$$

Taking the square of this expression we obtain

$$
W(u) e^{W(u)}=u,
$$

where we have set $W(u)=-2 \frac{\alpha^{2} L_{P l}^{2}}{\hbar^{2}}(\delta P)^{2}$ and $u=-\frac{\alpha^{2} L_{P l}^{2}}{2(\delta X)^{2}} e^{-2 \frac{\alpha^{2} L_{P 1}^{2}}{\hbar^{2}}\langle P\rangle^{2}}$.

The equation given by Eq.(9) is exactly the definition of the Lambert function [51]. The Lambert $W$ function is a multivalued functions. Its different branches are labelled by the integer $k=0, \pm 1, \pm 2, \cdots$. When $u$ is a real number Eq.(9) have two real solutions for $0 \geq u \geq-\frac{1}{e}$, denoted by $W_{0}(u)$ and $W_{-1}(u)$, or it can have only one real solution for $u \geq 0$, namely $W_{0}(u)$. For $-\infty<u<-\frac{1}{e}$, Eq.(9) have no real solutions.

Using Eq.(9) the uncertainty in momentum is then given by

$$
(\delta P)=\frac{\hbar e^{\frac{\alpha^{2} L_{P 1}^{2}}{\hbar^{2}}\langle P\rangle^{2}}}{2(\delta X)} \exp \left(-\frac{1}{2} W\left(-\frac{\alpha^{2} L_{P l}^{2} e^{\frac{2 \alpha^{2} L_{P l}^{2}}{\hbar^{2}}\langle P\rangle^{2}}}{2(\delta X)^{2}}\right)\right) .
$$


Then from the argument of the Lambert function in Eq.(10) we have the following condition

$$
\frac{\alpha^{2} L_{P l}^{2} e^{\frac{2 \alpha^{2} L_{P l}^{2}}{\hbar^{2}}\langle P\rangle^{2}}}{2(\delta X)^{2}} \leqslant \frac{1}{e}
$$

which leads to a minimal uncertainty in position given by

$$
(\delta X)_{\min }=\sqrt{\frac{e}{2}} \alpha L_{P l} e^{\frac{\alpha^{2} L_{P l}^{2}}{\hbar^{2}}\langle P\rangle^{2}} .
$$

The absolutely smallest uncertainty in position or minimal length is obtained for physical states for which we have $\langle P\rangle=0$ and $(\delta P)=\hbar /\left(\sqrt{2} \alpha L_{P 1}\right)$, and is given by

$$
(\delta X)_{0}=\sqrt{\frac{e}{2}} \alpha L_{P l}
$$

In terms of the minimal length the momentum uncertainty becomes

$$
(\delta P)=\frac{\hbar}{2(\delta X)} \exp \left(-\frac{1}{2} W\left(-\frac{1}{e}\left(\frac{(\delta X)_{0}}{(\delta X)}\right)^{2}\right)\right) .
$$

Here we observe that $\frac{1}{e} \frac{(\delta X)_{0}}{(\delta X)}<1$ is a small parameter, by virtue of the GUP*, and perturbative expansions to all orders in the Planck length can be safely performed.

Indeed a series expansion of Eq.(14) gives the corrections to the standard Heisenberg principle

$$
\delta P \simeq \frac{\hbar}{2(\delta X)}\left(1+\frac{1}{2 e}\left(\frac{(\delta X)_{0}}{(\delta X)}\right)^{2}+\frac{5}{8 e^{2}}\left(\frac{(\delta X)_{0}}{(\delta X)}\right)^{4}+\frac{49}{48 e^{3}}\left(\frac{(\delta X)_{0}}{(\delta X)}\right)^{6}+\ldots\right)
$$

This expression of $(\delta P)$ containing only odd powers of $(\delta X)$ is consistent with a recent analysis in which string theory and loop quantum gravity, considered as the most serious candidates for a theory of quantum gravity, put severe constraints on the possible forms of GUPs and MDRs [20].

Before ending this section and for later use let us recall the form of the GUP to leading order in the Planck length widely used in the literature on quantum gravity phenomenology. This GUP is given by

$$
(\delta X)(\delta P) \geq \frac{\hbar}{2}\left(1+\frac{\alpha^{2} L_{P l}^{2}}{\hbar^{2}}(\delta P)^{2}\right) .
$$

A simple calculation leads to the following minimal length

$$
(\delta X)_{0}=\alpha L_{P l}
$$

which is of order of the Planck length. However, as nicely noted in [41], this form of GUP do not fulfill the second requirement listed above. In the following sections we use the form of the GUP given by Eq.(14) and investigate the thermodynamics of the Schwarzschild black hole. We use units $\hbar=c=k_{\mathrm{B}}=1$ which imply $L_{P l}=M_{P l}^{-1}=T_{P l}^{-1}=\sqrt{G}$. 


\section{Black hole thermodynamics}

The metric of a four-dimensional Schwarzschild black hole is given by

$$
d s^{2}=\left(1-\frac{2 M G}{r}\right) d t^{2}-\left(1-\frac{2 M}{r}\right)^{-1} d r^{2}-r^{2} d \Omega^{2},
$$

where $M$ represents the mass of the black hole. The Schwarzschild horizon radius, located at $r_{h}$, is defined by

$$
r_{h}=2 M G \text {. }
$$

Near-horizon geometry considerations suggests to set $\delta X \simeq r_{h}$, and then Eq.(19) leads to minimum horizon radius and minimum mass given by

$$
r_{h}=(\delta X)_{0}=\sqrt{\frac{e}{2}} \alpha L_{P l}, \quad M_{0}=\frac{\alpha \sqrt{e}}{2 \sqrt{2}} M_{P l} .
$$

Therefore, black holes with mass smaller than $M_{0}$ do not exist.

In the standard Hawking picture, temperature and entropy of the Schwarzschild black hole of mass $M$ are $[52,53$

$$
T_{\mathrm{H}}=\frac{1}{8 \pi G M}, \quad S=4 \pi G M^{2} .
$$

Let us then examine the corrections to the above expressions due to the GUP*. Following the heuristic argument of Bekenstein we have

$$
T_{\mathrm{H}} \approx \frac{\delta P}{2 \pi}
$$

Using Eq.(14), the GUP*-corrected Hawking temperature is

$$
T_{\mathrm{H}}=\frac{1}{8 \pi M L_{P l}^{2}} \exp \left(-\frac{1}{2} W\left(-\frac{1}{e}\left(\frac{M_{0}}{M}\right)^{2}\right)\right) .
$$

On substituting Eq.(20) into Eq.(23) we obtain the following black hole maximum temperature

$$
T_{H}^{\max }=\frac{T_{P l}}{2 \pi \sqrt{2} \alpha} .
$$

The corrections to the standard Hawking temperature are obtained by expanding Eq.(23) in terms of $\frac{1}{e}\left(M_{0} / M\right)$. Indeed we obtain

$$
T_{\mathrm{H}} \simeq \frac{1}{8 \pi M L_{P l}^{2}}\left(1+\frac{1}{2 e}\left(\frac{M_{0}}{M}\right)^{2}+\frac{5}{8 e^{2}}\left(\frac{M_{0}}{M}\right)^{4}+\frac{49}{48 e^{3}}\left(\frac{M_{0}}{M}\right)^{6}+\ldots\right) .
$$


The variation of the Hawking temperature, Eq.(23), with the mass of the black hole is shown in figure 1.

It is interesting to inverse Eq.(23) and write the mass of the black hole as a function of the temperature

$$
M=\frac{1}{8 \pi T_{H} L_{P l}^{2}} \exp \left(\frac{1}{2}\left(\frac{T_{H}}{T_{H}^{\max }}\right)^{2}\right) .
$$

This relation shows that for temperatures larger than $T_{H}^{\max }$, the black hole mass increases with temperature. In our framework, such a behavior is forbidden by the cut-off brought by GUP* However, in the noncommutative approach to radiating black hole, this behavior is allowed because of a lack of a generalized uncertainty principle [50].

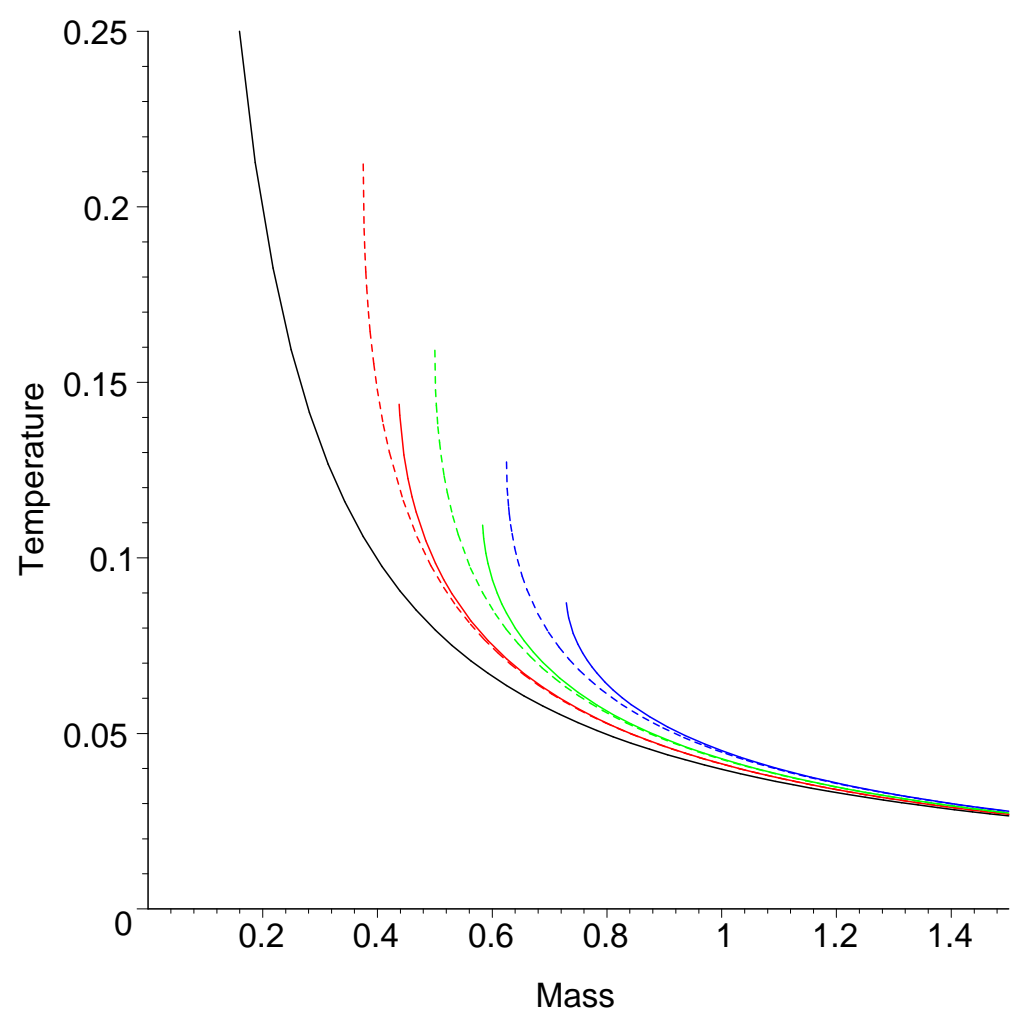

Figure 1: The temperature versus BH mass. From left to right: the Hawking result (black solid line), GUP (doted line) and GUP* results (solid line) for $\alpha=0.75$ (red), $\alpha=1$ (green), $\alpha=1.25$ (blue) respectively.

We turn now to the calculation of the micro canonical entropy of a large black hole. In the standard situation the entropy is proportional to the black hole horizon-area. Following heuristic considerations due to Bekenstein, the minimum increase of the area of a black hole absorbing a classical particle of energy $E$ and size $R$ is given by $(\Delta A)_{0} \simeq 4 L_{P l}^{2}(\ln 2) E R$. At the quantum mechanical level the size and the energy of the particle are constrained to verify $R \sim 2 \delta X$ and $E \sim \delta P$. Then we have $(\Delta A)_{0} \simeq 8 L_{P l}^{2}(\ln 2) \delta X \delta P$. 
Extending this approach to the case with $\mathrm{GUP}^{*}$ and using near horizon geometry considerations, we obtain

$$
(\Delta A)_{0} \approx 4 L_{P l}^{2} \ln 2 \exp \left(-\frac{1}{2} W\left(-\frac{1}{e} \frac{A_{0}}{A}\right)\right),
$$

where $A=4 \pi(\delta X)^{2}$ and $A_{0}=4 \pi(\delta X)_{0}^{2}$ are respectively the horizon area and minimum horizon area of the black hole. With the aid of the Bekenstein calibration factor for the minimum increase of entropy $(\Delta S)_{0}=\ln 2$ we have

$$
\frac{d S}{d A} \simeq \frac{(\Delta S)_{0}}{(\Delta A)_{0}}=\frac{1}{4 L_{P l}^{2}} \exp \left(\frac{1}{2} W\left(-\frac{1}{e} \frac{A_{0}}{A}\right)\right) .
$$

Before integrating over $A$ we note that the existence of a minimum horizon area enforces us to set the lower limit of integration as $A_{0}$. Then the entropy, up to a irrelevant constant, is

$$
S \simeq \frac{1}{4 L_{P l}^{2}} \int_{A_{0}}^{A} \exp \left(\frac{1}{2} W\left(-\frac{1}{e} \frac{A_{0}}{A}\right)\right) d A . .
$$

The relation $e^{\frac{W(x)}{2}}=\sqrt{x / W(x)}$ allows us to write Eq.(29) as

$$
S=\frac{A_{0}}{4 e L_{P l}^{2}} \mathbf{P V} \int_{-\frac{1}{e}}^{-\frac{1}{e} \frac{A_{0}}{A}} y^{-\frac{3}{2}}[W(y)]^{-\frac{1}{2}} d y
$$

where $\mathbf{P V}$ means the Cauchy principal value of the integral. Setting $y=-\frac{1}{e} \frac{A_{0}}{A}$ and performing the integration we obtain the $\mathrm{GUP}^{*}$-corrected black hole entropy

$$
S=\frac{A_{0}}{8 e L_{P l}^{2}}\left\{\operatorname{Ei}\left(-\frac{1}{2} W\left(-\frac{1}{e} \frac{A_{0}}{A}\right)\right)-2\left(-\frac{1}{e} \frac{A_{0}}{A} W\left(-\frac{1}{e} \frac{A_{0}}{A}\right)\right)^{-\frac{1}{2}}-2 \sqrt{e}-\operatorname{Ei}\left(\frac{1}{2}\right)\right\}
$$

where $\operatorname{Ei}(x)$ is the exponential function.

Expanding Eq.(31) in the parameter $\frac{1}{e}\left(A_{0} / A\right)$ we have

$$
S=\left\{\frac{A}{4 L_{P l}^{2}}-\frac{A_{0}}{8 L_{P l}^{2} e} \ln \frac{A}{A_{0}}+\frac{3 \pi \alpha^{2}}{16 e}\left(\frac{A_{0}}{A}\right)+\frac{25 \pi \alpha^{2}}{192 e^{2}}\left(\frac{A_{0}}{A}\right)^{2}+\frac{343 \pi \alpha^{2}}{2304 e^{3}}\left(\frac{A_{0}}{A}\right)^{3}+\ldots+\mathrm{C}\right\}
$$

where the constant is given by

$$
\mathrm{C}=\frac{A_{0}}{8 L_{P l}^{2} e}\left\{\gamma-1-2 \ln (2 e)-2 \sqrt{e}-\operatorname{Ei}\left(\frac{1}{2}\right)\right\} \simeq-4.60 \frac{\alpha^{2}}{L_{P l}^{2}}
$$

and $\gamma$ is the Euler constant. The dependence on the Planck length is contained in $A_{0} \sim L_{P l}^{2}$. We observe that we have reproduced, in our framework with GUP*, the log-area correction with a negative sign. Other approaches like string theory, loop quantum gravity and effectif 
models with GUPs and/or MDRs, lead to the same sub-leading logarithmic correction. Setting $\rho=-\frac{\pi \alpha^{2}}{4}$ and $\beta=\frac{3 \pi^{2} \alpha^{4}}{8}$ in Eqs. (25) and (32) we obtain

$$
\begin{aligned}
T_{\mathrm{H}} & =\frac{M_{P l}^{2}}{8 \pi M}\left[1-\frac{\rho}{4 \pi}\left(\frac{M_{P l}}{M}\right)^{2}+\frac{\rho^{2}+\beta / 4}{16 \pi^{2}}\left(\frac{M_{P l}}{M}\right)^{4}\right], \\
S & =\frac{A}{4 L_{P l}^{2}}+\rho \ln \frac{A}{L_{P l}^{2}}+\frac{\beta L_{P l}^{2}}{A} .
\end{aligned}
$$

These expressions are exactly the temperature and entropy obtained in loop quantum gravity and string theory approach quantum gravity.

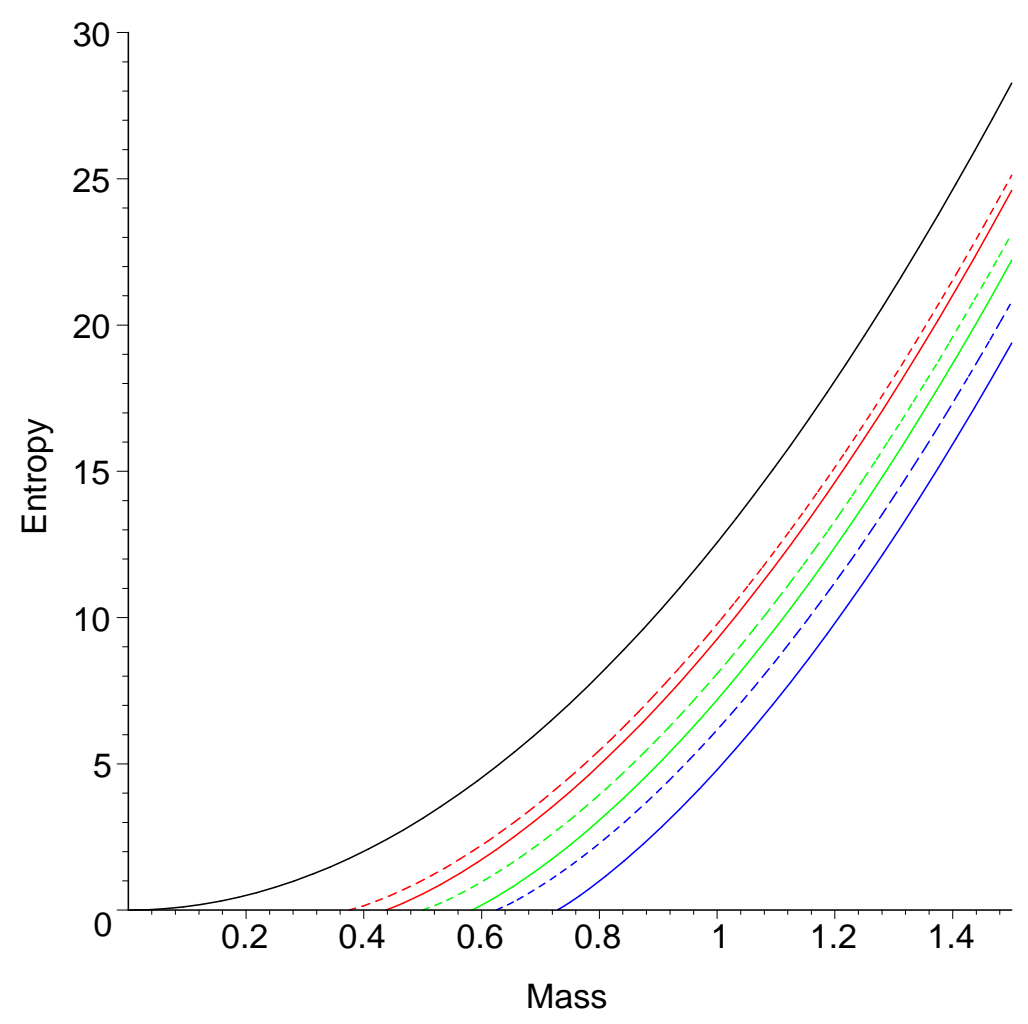

Figure 2: The entropy versus BH mass. From left to right: the Hawking result (black solid line), GUP (doted line) and GUP* results (solid line) for $\alpha=0.75$ (red), $\alpha=1$ (green) and $\alpha=1.25$ (blue) respectively.

From figures 1 and 2 it follows that the GUP*-corrected temperature and entropy are respectively higher and smaller than the semi classical results.

\section{Black holes evaporation}

As a warming to study the Hawking radiation process of the Schwarzschild black hole, we examine the effects of GUP* on the black body radiation spectrum. With the aid of the 
squeezed momentum measure given by Eq.(5), which suppress the contribution of unwanted high momenta, the energy density of a black body at temperature $T$ is defined by

$$
\mathcal{E}_{\gamma}=2 \int d^{3} p e^{-\alpha^{2} L_{P l}^{2} p^{2}} \frac{p}{e^{\frac{p}{T}}-1} .
$$

Using the variable $y=\beta p(\beta=1 / T)$ and expanding the exponential, equation (36) becomes

$$
\mathcal{E}_{\gamma}=8 \pi T^{4} \sum_{n=0}^{\infty} \frac{(-1)^{n}}{n !}\left(\alpha T / T_{P l}\right)^{2 n} \int_{0}^{\infty} d y \frac{y^{2 n+3}}{e^{y}-1} .
$$

Now with the help of the following definition of the Riemann zeta function

$$
\int_{0}^{\infty} d y \frac{y^{s-1}}{e^{y}-1}=\Gamma(s) \zeta(s)
$$

we obtain

$$
\mathcal{E}_{\gamma}=8 \pi T^{4} \sum_{n=0}^{\infty} \frac{(-1)^{n}}{n !}\left(\alpha T / T_{P l}\right)^{2 n} \Gamma(2 n+4) \zeta(2 n+4) .
$$

This energy density is defined only for values of temperatures below some characteristic scale. In fact Eq.(39) is an alternating series which converge if and only if

$$
\lim _{n \rightarrow \infty}\left[\frac{1}{n !}\left(\alpha T / T_{P l}\right)^{2 n} \Gamma(2 n+4) \zeta(2 n+4)\right]=0 .
$$

From this relation it follows that

$$
T<\alpha^{-1} T_{P l}
$$

as expected from the Gaussian damping factor in Eq.(36). However, we note that we have a stronger condition on $T$. Indeed in our framework, the maximum temperature of the black hole is given by Eq.(24) and it is approximately $0.1 T_{P l}$ for $\alpha$ of order one. Then the condition on the $\mathrm{BH}$ temperature is rewritten as $T / T_{P l}<0.1$. For our purpose, the latter constraint allows us to cut the series in Eq.(39) at $n=1$. Using $\zeta(4)=\frac{\pi^{4}}{90}$ and $\zeta(6)=\frac{\pi^{6}}{945}$ and Eq.(24) we finally obtain, from Eq.(39), the following expression

$$
\mathcal{E}_{\gamma}(T)=\frac{8 \pi^{5}}{15} T^{4}\left(1-\frac{15}{63}\left(\frac{T}{T_{H}^{\max }}\right)^{2}\right) .
$$

The first term is the standard Stefan-Boltzmann law while the second term is the correction brought by GUP*.

We are now ready to study the Hawking evaporation process. The intensity emitted by a black hole of mass $M$ is defined by

$$
I=A \mathcal{E}_{\gamma}\left(T_{\mathrm{H}}\right)
$$


where $A$ is the $\mathrm{BH}$ horizon area. Invoking energy conservation, the evaporation rate of the black hole is

$$
\frac{d M}{d t}=-A \mathcal{E}_{\gamma}\left(T_{\mathrm{H}}\right)
$$

Using Eq. (23) for the corrected Hawking temperature we obtain

$$
\frac{d M}{d t}=-\frac{\gamma_{1}}{M^{2} L_{P l}^{4}} \exp \left(-2 W\left(-\frac{1}{e}\left(\frac{M_{0}}{M}\right)^{2}\right)\right)\left(1-\frac{8 \gamma_{2}}{e \gamma_{1}}\left(\frac{M_{0}}{M}\right)^{2} \exp \left(-W\left(-\frac{1}{e}\left(\frac{M_{0}}{M}\right)^{2}\right)\right)\right),
$$

with $\gamma_{1}=\frac{\pi^{2}}{480}, \gamma_{2}=\frac{\pi^{2}}{16128}$. The deviations from the standard expression are obtained by applying a series expansion in $\frac{1}{e}\left(M_{0} / M\right)$

$\frac{d M}{d t}=-\frac{\gamma_{1}}{M^{2} L_{P l}^{4}}\left(1+\frac{2}{e}\left(\frac{M_{0}}{M}\right)^{2}+\frac{4}{e^{2}}\left(1-\frac{2 \gamma_{2}}{e \gamma_{1}}\right)\left(\frac{M_{0}}{M}\right)^{4}+\frac{25}{3 e^{3}}\left(1-\frac{72 \gamma_{2}}{25 e \gamma_{1}}\right)\left(\frac{M_{0}}{M}\right)^{6}+\ldots\right)$.

The variation of the evaporation rate with the black hole mass is shown in Figure 3. We clearly observe that the divergence for $M \rightarrow 0$ in the standard description of the black hole evaporation process is now completely regularized by the GUP*. This regularization is also reflected by the constraint (41), which suppress the evaporation process beyond the Planck temperature. This phenomenon is similar to the prevention, by the standard uncertainty principle, of the hydrogen atom from total collapse. In our picture, the regularization can be considered as a dynamical effect and not as a consequence of any quantum symmetry in the theory.

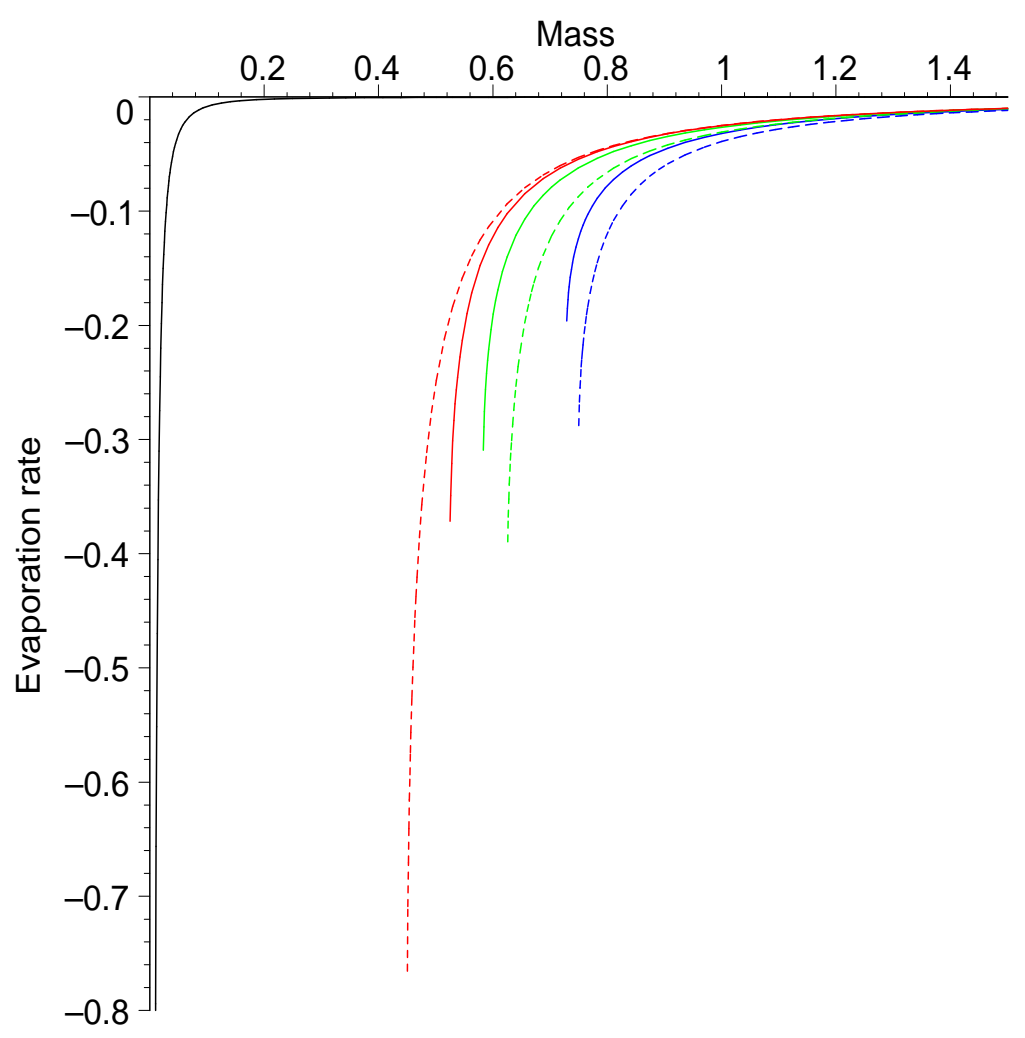


Figure 3: The evaporation rate versus BH mass. From left to right: the Hawking result (black solid line), GUP (doted line) and GUP* results (solid line) for $\alpha=0.90$ (red), $\alpha=1$. (green) and $\alpha=1.25$ (blue).

On the other hand, we observe that the evaporation phase ends when the BH mass becomes equal to $M_{0}$ with a minimum rate given by

$$
\left(\frac{d M}{d t}\right)_{\min }=-\frac{e^{2}}{M_{0}^{2}}\left(\gamma_{1}-8 \gamma_{2}\right) M_{P l}^{4}
$$

Thus the evaporation process of a black hole with initial mass $M>M_{0}$ continue until the horizon radius becomes $(\delta X)_{0}$, leaving a massive relic referred to, in the literature, as a black hole remnant (BHR). To find the nature of the BHR we calculate the heat capacity defined by

$$
C=\frac{d M}{d T_{\mathrm{H}}}
$$

Using the expression of temperature given by (23) we easily obtain

$$
C=-8 \pi M^{2} L_{P l}^{2}\left(1+W\left(-\frac{1}{e}\left(\frac{M_{0}}{M}\right)^{2}\right)\right) \exp \left(\frac{1}{2} W\left(-\frac{1}{e}\left(\frac{M_{0}}{M}\right)^{2}\right)\right) .
$$

This expression vanishes when $1+W\left(-\frac{1}{e}\left(M_{0} / M\right)^{2}\right)=0$, whose solution is $M=M_{0}$. We conclude that the heat capacity of the black hole vanishes at the end point of the evaporation process characterized by a BHR with mass $M_{0}$. Besides the gravitational interaction with the surrounding, the vanishing of the heat capacity reveals the inert character of the BHRs and thus make them as potential candidates to explain the origin of dark matter [18, 19]. Finally we note that, as it is the case with the form of the GUP to leading order in the Planck length, the BHRs are also a consequence of GUP* [17, 55].

We have drawn the variation of the heat capacity with $\mathrm{BH}$ mass in figure 4 . In it we see that, the heat capacity vanishes for $M_{0} \simeq 0.50,0.75$ in the case with GUP and $M_{0} \simeq 0.58,0.87$ in the case with GUP*. 


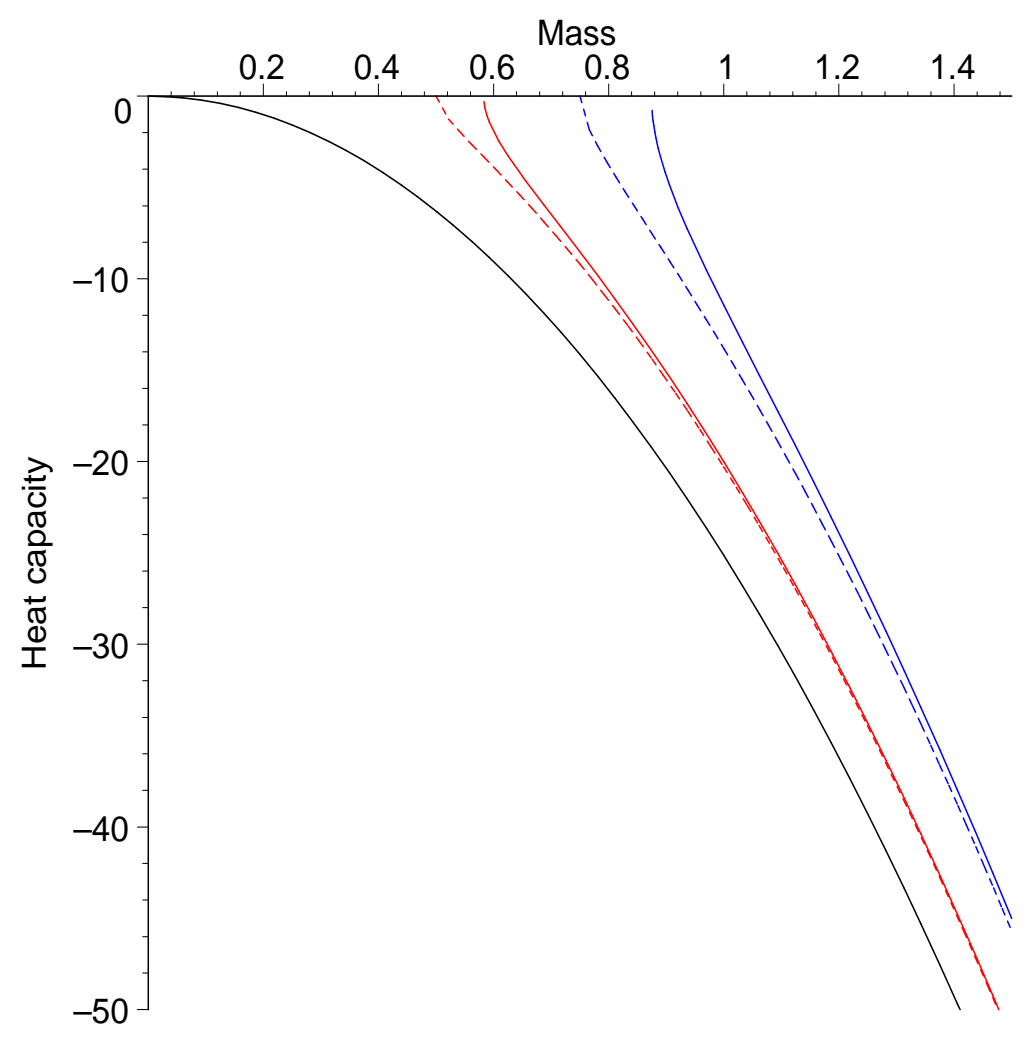

Figure 4: The heat capacity versus BH mass. From left to right: the standard result (black solid line), GUP (doted line) and GUP* results (solid line) for $\alpha=1$ (red) and $\alpha=1.5$ (blue) respectively.

Taylor expanding Eq.(49) we have

$$
C=-8 \pi M^{2} L_{P l}^{2}\left(1-\frac{3}{2 e}\left(\frac{M_{0}}{M}\right)^{2}-\frac{7}{8 e^{2}}\left(\frac{M_{0}}{M}\right)^{4}-\frac{55}{48 e^{3}}\left(\frac{M_{0}}{M}\right)^{6}-\ldots\right) .
$$

The standard expression of the heat capacity $C=-8 \pi M^{2}$ is reproduced in the limit of black holes with mass larger than the minimum mass $M_{0}$. The correction terms to the heat capacity due to $\mathrm{GUP}^{*}$ are all positive indicating that the evaporation process is accelerated and leading to a corrected decay time smaller than the decay time in the standard case.

Let us consider a black hole starting the evaporation process with a mass $M$ and ending the process with the minimum mass $M_{0}$. Using (45) and the variable $y=-\frac{1}{e}\left(M_{0} / M\right)^{2}$, the decay time is given by

$$
\begin{aligned}
t & =(-1)^{7 / 2} \frac{M_{0}^{3} L_{P l}^{4}}{2 \gamma_{1} e^{3 / 2}} \int_{-\frac{1}{e}\left(M_{0} / M\right)^{2}}^{-\frac{1}{e}} W^{-5 / 2}(y) e^{-\frac{1}{2} W(y)} d y \\
& +(-1)^{5 / 2} \frac{\gamma_{2}}{2 \gamma_{1}^{2} e^{1 / 2}} M_{0} \alpha^{2} L_{P l}^{2} \int_{-\frac{1}{e}\left(M_{0} / M\right)^{2}}^{-\frac{1}{e}} W^{-3 / 2}(y) e^{-\frac{1}{2} W(y)} d y
\end{aligned}
$$


Performing the integration we obtain

$$
t=\frac{M_{0}^{3} L_{P l}^{4}}{3 \gamma_{1} e^{3 / 2}}\left[4(1-\epsilon) \frac{\sqrt{-y}}{W(y)}-\sqrt{8 \pi}\left(1-\frac{\epsilon}{2}\right) \operatorname{erf}\left(\sqrt{-\frac{W(y)}{2}}\right)+\frac{\sqrt{-y}}{W^{2}(y)}\right]+C,
$$

where the constant $C$ is the value for $y=-1 / e$ and $\epsilon=\frac{3 \gamma_{2}}{\gamma_{1}} e \alpha^{2}\left(\frac{M_{P l}}{M_{0}}\right)^{2} \sim 10^{-6}$ for $\alpha$ of order one. Ignoring $\epsilon$ and performing a series expansion in $y$ we have

$$
t=\frac{M^{3} L_{P l}^{4}}{3 \gamma_{1}}\left(1-\frac{6}{e}\left(\frac{M_{0}}{M}\right)^{2}-\frac{2}{e^{2}}\left(\frac{M_{0}}{M}\right)^{4}+\frac{1}{3 e^{3}}\left(\frac{M_{0}}{M}\right)^{6}+\ldots\right)
$$

Then to first order in $\frac{1}{e}\left(M_{0} / M\right)$ the relative correction to the decay time is

$$
\frac{\Delta t}{t_{0}}=-\frac{6}{e}\left(\frac{M_{0}}{M}\right)^{2}
$$

where $t_{0}=\frac{M^{3} L_{P l}^{4}}{3 \gamma_{1}}$ is the decay time without GUP*. From Eq.(154), it follows that black holes with GUP* are hotter and decay faster than in the standard case.

Let us now turn to a comparison of the corrected BH thermodynamics with GUP* with the corrections brought by the GUP to leading order in the Planck length. Since our comparison is quantitative we use the Planck units. Repeating the same calculations as above with the GUP given by Eq.(16), the temperature, the entropy and the heat capacity of the black hole are respectively given by

$$
\begin{gathered}
T_{G U P}=\frac{M}{\pi \alpha^{2}}\left(1-\sqrt{1-\frac{\alpha^{2}}{4 M^{2}}}\right) \\
S_{G U P}=2 \pi M^{2}\left(1+\sqrt{1-\frac{\alpha^{2}}{4 M^{2}}}\right)-\frac{\pi \alpha^{2}}{8} \ln \left(\frac{8 M^{2}}{\alpha^{2}}\left(1+\sqrt{1-\frac{\alpha^{2}}{4 M^{2}}}\right)-1\right)-\frac{\pi \alpha^{2}}{8}
\end{gathered}
$$

and

$$
C_{G U P}=\pi \alpha^{2} \frac{\sqrt{1-\frac{\alpha^{2}}{4 M^{2}}}}{\sqrt{1-\frac{\alpha^{2}}{4 M^{2}}}-1} .
$$

The minimum black hole mass and maximum temperature allowed by GUP are $M_{0}=(\delta X)_{0} / 2=$ $\frac{\alpha}{2}$ and $T_{\max }=1 / 2 \pi \alpha$. In figures $1,2,3$ and 4 we have plotted, besides the results obtained with $\mathrm{GUP}^{*}$, the variation of temperature, entropy, evaporation rate and heat capacity with GUP as functions of the black hole mass for different values of the parameter $\alpha$. Figure 2 shows, that in the scenario with GUP*, the BH entropy decreases compared to the entropy in the standard case and the scenario with GUP. This reveals the deeper quantum nature of the black hole 
in the scenario with GUP*. Thus quantum effects become manifest at an earlier stage of the evaporation phase than was predicted by the semi classical Hawking analysis [54] and the GUP analysis [55].

The calculation of the evaporation rate in the framework with GUP requires a careful analysis. In all the calculations done until now, the validity of the Stefan-Boltzmann law is assumed, ignoring the UV cut-off implemented by GUP. However, it was pointed in [16] that the effect of the GUP should be also reflected in a modification of the de Broglie wave length relation

$$
\lambda=\frac{1}{2 p}\left(1+\alpha^{2} p^{2}\right) .
$$

This relation must be translated into a modification of the momentum measure such that the contributions of high momenta are suppressed. As shown in [7], the GUP to leading order in the Planck length leads to a squeezing of the momentum measure by a factor $\frac{1}{\left(1+\alpha^{2} L_{P l}^{2} p^{2}\right)}$. Then following the same calculation leading to Eq.(39), the energy density of a black body with GUP is

$$
\mathcal{E}_{\gamma}=2 \int \frac{d p^{3}}{\left(1+\alpha^{2} L_{P l}^{2} p^{2}\right)} \frac{p}{e^{\frac{p}{T}}-1} .
$$

Performing the integral and using the same argument as before, we obtain the expression given by Eq. (42). We note, that in a recent calculation of the Stefan-Boltzmann law with GUP [24], the sign of the correction term is positive, in contradiction with the role of the UV cut-off implemented by the GUP.

The correct evaporation rate with GUP is then given by

$$
\left(\frac{d M}{d t}\right)_{G U P}=-\frac{128 \pi^{2} M^{6}}{15 \alpha^{8}}\left(1-\sqrt{1-\frac{\alpha^{2}}{4 M^{2}}}\right)^{4}+\frac{1024 \pi^{2} M^{8}}{63 \alpha^{10}}\left(1-\sqrt{1-\frac{\alpha^{2}}{4 M^{2}}}\right)^{6} .
$$

In figure 3 we observe that the evaporation process with GUP is retarded compared to the process with $\mathrm{GUP}^{*}$ and that the process ends at a mass $M_{0}=\alpha / 2$ with a minimum rate given by

$$
\left(\frac{d M}{d t}\right)_{\min , G U P}=-\frac{32 \pi^{2}}{1260 M_{0}^{2}}
$$

which is greater (in absolute value) than the one obtained with GUP*.

In table 1 we show the GUP and GUP*-corrected thermodynamics of two black holes with initial mass equal to $2 M_{P l}$ and $5 M_{P l}$ for $\alpha=1$. The first row gives the semi classical Hawking results. The second row gives the GUP-corrected results and the third row the GUP* - corrected ones. It is interesting to note that, in the scenario with GUP*, the final stage of the evaporation phase is a remnant with a mass larger than the one obtained with GUP and that the decay time 
is drastically reduced. In a scenario with extra dimensions, these results may have important consequences on possible black holes production at particle colliders and in ultrahigh energy cosmic ray (UHECR) air-showers.

Finally, let us notice that the corrections to the black hole thermodynamics become indistinguishable in the two version of GUP in the limit of large mass and small values of $\alpha$. However, for growing values of the parameter $\alpha$, corresponding to strong gravitational effects, the predictions of the two GUPs concerning the entropy become different even for massive black holes.

Table 1. GUP and GUP*-corrected thermodynamics for two BHs with mass $M=2$ and $M=5$ (in Planck units). The deviations from the Hawking results are also given.

$M=2$

\begin{tabular}{|c|c|c|c|c|c|}
\hline$\alpha$ & Minimum mass & Initial temp & Final temp & Decay time & Entropy \\
\hline 0 & - & 0.019 & $\infty$ & 129.69 & 50.27 \\
\hline 1.0 & 0.5 & $0.020(+3 \%)$ & 0.16 & $111.92(-14 \%)$ & $44.66(-11 \%)$ \\
\hline 1.0 & $0.58(+16 \%)$ & $0.020(+3 \%)$ & $0.11(-31 \%)$ & $3.33(-97 \%)$ & $43.73(-13 \%)$ \\
\hline
\end{tabular}

$M=5$

\begin{tabular}{|c|c|c|c|c|c|}
\hline$\alpha$ & Minimum mass & Initial temp & Final temp & Decay time & Entropy \\
\hline 0 & - & 0.008 & $\infty$ & 2026.42 & 314.16 \\
\hline 1.0 & 0.5 & 0.008 & 0.16 & $1976.60(-2.5 \%)$ & $307.10(-2 \%)$ \\
\hline 1.0 & $0.58(+16 \%)$ & 0.008 & $0.11(-31 \%)$ & $22.17(-99 \%)$ & $306.18(-2.2 \%)$ \\
\hline
\end{tabular}

\section{Conclusion}

In this paper we have studied how black holes thermodynamic parameters are affected by a GUP to all orders in the Planck length. We have obtained exact analytic expressions for the Hawking temperature and entropy. Particularly we found that a black hole with a mass smaller than a minimum mass do not exist. The existence of a energy scale which is one order below the Planck scale allowed us to calculate, to leading order, the deviations from the standard StefanBoltzmann law. Then we investigated the Hawking radiation process of the Schwarzschild black hole and shown that at the end of the evaporation phase a inert massive relic continue to exist as a black hole remnant (BHR) with zero entropy, zero heat capacity and non zero finite temperature. For completeness, we have also compared our results with the semi classical results and the predictions of the GUP to leading order in the Planck length. In particular, we have shown that the entropy in our framework is smaller than the entropy in the standard case and with GUP. We have also made the correct calculation of the evaporation rate with GUP. Finally, we have shown that black holes with the form of GUP used in this paper are 
hotter, shorter-lived and tend to evaporate less than black holes in the semi classical and the GUP to leading order pictures. On the other hand, we have found that the predictions of the GUP to all orders in the Planck length and the GUP to leading order in the Planck length, concerning the entropy, become different for strong gravitational effects and large black holes mass, suggesting a further investigation of the early universe thermodynamics in the framework

with the GUP to all orders in the Planck length. In a future work we will examine the effects of the GUP to all orders in the Planck length on black holes thermodynamics in a scenario with extra dimensions.

\section{Acknowledgments}

The author thanks the referees for their comments and valuable remarks.

\section{References}

[1] G. Veneziano, Europhys. Lett. 2, 199 (1986).

[2] E. Witten, Phys. Today, Apr. 24 (1996).

[3] D. Amati, M. Ciafaloni and G. Veneziano, Phys. Lett. B 216, 41 (1989); Nucl. Phys. B 347, 550 (1990); Nucl. Phys. B403, 707 (1993).

[4] K. Konishi, G. Paffuti and P. Provero, Phys. Lett. B 234, 276 (1990).

[5] L. J. Garay, Int. J. Mod. Phys. A 10, 145 (1995).

[6] M. Maggiore, Phys. Rev. D 49, 5182 (1994) hep-th/9305163]; Phys. Lett. B 319, 83(1993) hep-th/9309034.

[7] A. Kempf, G. Mangano and R.B. Mann, Phys. Rev. D 52, 1108 (1995) hep-th/9412167.

[8] A. Kempf and G.Mangano, Phys. Rev. D55, 7909 (1997).

[9] M. Maggiore, Phys. Lett. B 304, 65 (1993) hep-th/9301067.

[10] F. Scardigli, Phys. Lett. B 452, 39 (1999) hep-th/9904025.

[11] B. Bolen and M. Cavaglia, Gen. Rel. Grav. 37, 1255 (2005) [gr-qc/0411086].

[12] A. J. M. Medved and E. C. Vagenas, Phys.Rev. D 70, 1240(2004).

[13] A. J. M. Medved, Class.Quant.Grav. 22, 133(2005). 
[14] G. Gour and A. J. M. Medved, Class. Quant. Grav. 20, 3307(2003).

[15] K. Nozari and T. Azizi, Int. J. Quant. Inf. 3, 623(2005) [gr-qc/0504090].

[16] G. A. Amelino-Camelia, M. Arzano, Y. Ling and G. Mandanici, Class. Quant. Grav. 23, 2585 (2006) [gr-qc/0506110].

[17] R. J. Adler, P. Chen, D. I. Santiago, Gen. Rel. Grav. 33, 2101 (2001) [arXiv: gr-qc/0106080.

[18] P. Chen, New Astron. Rev. 49, 233(2005).

[19] P. Chen and R. J. Adler, Nucl. Phys. Proc. Suppl. 124, 103 (2003)[gr-qc/0205106].

[20] K. Nozari and A. S. Sefiedgar, Phys. Lett. B 635, 156(2006) [gr-qc/0601116].

[21] A. J. M. Medved and E. C. Vagenas, Phys. Rev. D 70 , 124021(2004).

[22] A. Chatterjee and P. Majumdar, Phys. Rev. Lett. 92, 141301(2004).

[23] R. K. Kaul and P. Majumdar, Phys. Rev. Lett. 84, 5255 (2000).

[24] G. A. Camellia, M. Arzano and A. Procaccini, Phys. Rev. D 70, 107501(2004).

[25] A. Chatterjee and P. Majumdar, Phys. Rev. D 71, 024003(2005).

[26] Y. S. Myung, Phys. Lett. B 579, 205(2004).

[27] M. M. Akbar and S. Das, Class. Quant. Grav. 21, 1383(2004).

[28] S. Das, Class. Quant. Grav. 19, 2355 (2002).

[29] S. Hod, Class. Quant. Grav. 21, L97 (2004) .

[30] A. J. M. Medved, Class. Quant. Grav. 22, 133 (2005).

[31] A. J. M. Medved, Class. Quant. Grav. 22, 5195 (2005).

[32] A. J. M. Medved and E. C. Vagenas, Mod. Phys. Lett. A 20, 1723 (2005).

[33] G. Gour and A. J. M. Medved, Class. Quant. Grav. 20, 3307(2003).

[34] L. Alejandro Correa-Borbonet, Braz. J. Phys. 35, 1145(2005).

[35] Y. Ko, S. Lee and S. Nam, Tests of Quantum Gravity via Generalized Uncertainty Principle, [hep-th/0608016 v2]. 
[36] http://lhc-new-homepage.web.cern.ch/lhc-new-homepage/

[37] http://www.fnal.gov/projects/muon collider/

[38] J. L. Feng and A. D. Shapere, Phys. Rev. Lett. 88, 021303 (2002) hep-ph/0109106]; A. Ringwald and H. Tu, Phys. Lett. B 525, 135 (2002) hep-ph/0111042 ; Phys. Lett. B 529, 1 (2002) hep-ph/0201139]; M. Ave, E. J. Ahn, M. Cavagli‘a and A. V. Olinto, astro-ph/0306344 Phys. Rev. D 68, 14043004 (2003) hep-ph/0306008; S. I. Dutta, M. H. Reno and I. Sarcevic, Phys. Rev. D 66, 033002 (2002) [hep-ph/0204218]. R. Emparan, M. Masip and R. Rattazzi, Phys. Rev. D 65, 064023 (2002) hep-ph/0109287]; A. Mironov, A. Morozov and T. N. Tomaras, hep-ph/0311318; P. Jain, S. Kar, S. Panda and J. P. Ralston, Int. J. Mod. Phys. D 12, 1593 (2003) [hep- ph/0201232].

[39] M. Cavagli‘a, Int. J. Mod. Phys. A 18, 1843 (2003) hep-ph/0210296]. G. Landsberg, Eur. Phys. J. C 33, S927 (2004) hep-ex/0310034; P. Kanti, Int. J. Mod. Phys. A 19, 4899 (2004) hep-ph/0402168.

[40] G. Amelino-Camelia, Introduction to Quantum-Gravity Phenomenology, the 40th Karpacz Winter School in Theoretical Physics gr-qc/0412136.

[41] S. Hossenfelder, Phys. Rev. D 73, 105013 (2006) hep-th/0603032;Class. Quant. Grav. 23, 1815 (2006) hep-th/0510245.

[42] H. S. Snyder, Phys. Rev. 71, 38 (1947).

[43] A. Connes, Noncommutative geometry, Academic Press, 1994.

[44] J. Madore, An introduction to noncommutative geometry and its physical applications, Cambridge University Press, 1999.

[45] G. Landi, An introduction to noncommutative spaces and their geometries, Springer Verlag, 1997.

[46] J.M. Gracia-Bondia, J.C. Varilly and H. Figuera, Elements of noncommutative geometry, Birkhäuser, 2001.

[47] A. Smailagic, E. Spallucci, J. Phys. A 36, L467 (2003); A. Smailagic, E. Spallucci, J. Phys. A 36, L517 (2003); A. Smailagic, E. Spallucci, J. Phys. A 37, 7169 (2004).

[48] J. Moffat, Phys. Lett. B 506, 193 (2001) hep-th/0011035.

[49] Kh. Nouicer, M. Debbabi, Phys. Lett. A 361, 305 (2007). 
[50] P. Nicolini, J. Phys. A 38, L631 (2005); P. Nicolini, A. Smailagic, E. Spallucci Phys. Lett. B 632, 547, (2006).

[51] R.M. Corless, G.H. Gonnet, D.E. Hare, D.J. Je rey and D.E. Knuth, Adv. Comput. Math. 5, 329 (1996)

[52] S. Hawking, Commun. Math. Phys. 43, 199 (1975).

[53] J. D. Bekenstein, Phys. Rev. D 7, 2333 (1973); Lett. al Nuovo Cimento 4, 737 (1972).

[54] M. Cavaglià, Int. J. Mod. Phys. A 18, 1843 (2003).

[55] M. Cavaglià and S. Das, Class. Quant. Grav. 21, 4511 (2004) [hep-th/0404050] . 